\title{
Conquérir la négritude \\ Considérations inessentielles sur le genre noir
}

\author{
Alexandra Alekseeva, Fabien Schang \\ Département de Français, Faculté des sciences humaines, \\ Université de Johannesburg
}

Quel message est apporté par le courant littéraire de la négritude, et comment procède-t-il pour le transmettre? C'est par le biais d'une écriture introspective que la diaspora noire a conquis sa dignité et dépassé le stade victimaire, par-delà le seul cadre de la communauté francophone. A travers l'histoire de la traite et de la colonisation, notre lecture procédera en trois phases: une phase locutoire, consacrée à un rappel chronologique du contexte noir dans l'Histoire; une phase illocutoire, où seront exposées les différentes réactions de l'intelligentsia noire face à la ségrégation et au passif colonial; une phase perlocutoire, qui conclura l'étude en observant l'impact de la négritude sur la réalité sociale du genre noir. Au-delà du courant historique francophone, l'idée de négritude incarne le problème des constructions d'identité et illustre parfaitement la difficulté de la condition humaine: être ce que nous voulons de ce que les autres veulent faire de nous.

Mots-clefs: essentialisme, études de genre, identité, nationalisme noir, négritude

\section{Conquering "la négritude" Inessential considerations on the black gender}

\author{
Alexandra Alekseeva, Fabien Schang \\ Associate Researchers at French Department \\ Faculty of the Humanities, University of Johannesburg
}

Which message is conveyed by the literary trend of the so-called négritude, and how does it proceed to do so? The black diaspora conquered dignity and went beyond the stage of victimhood by means of an introspective script. Throughout the history of slave trade and colonization, our reading runs into three main steps, namely: a locutionary step, devoted to a chronological reminder of the black context in History; an illocutionary step, where the various reactions of black intelligentsia to segregation and colonial liabilities will be surveyed; a perlocutionary step, which concludes the investigation by observing the effects of "négritude" on the social reality of the black gender. Beyond the French-speaking historical trend, the view of "négritude" embeds the problem of constructing identities and relevantly exemplifies the difficulty of human condition, that is: being what one wants to be from what the others want us to be.

Keywords: black nationalism, essentialism, gender studies, identity, “négritude”. 


\section{Introduction : l'esprit ou la lettre}

Quelques mois avant les élections présidentielles françaises de 2007, la candidate socialiste Ségolène Royal voulut donner de la hauteur à sa campagne via une ascension de la muraille de Chine. Après la roche de Solutré du modèle Mitterrand, la jeune élève fit mieux que son maître en matière d'innovation linguistique lorsqu'elle s'essaya à une citation du cru: «Comme le disent les Chinois: qui n'est pas venu sur la Grande muraille n'est pas un brave, et qui vient sur la Grande muraille conquiert la bravitude $»$.

"Bravitude", ou "bravoure"? Génie, ou abus du langage? Question mesquine, ou question de principe? La leçon la plus intéressante de cet épisode tragi-comique vint davantage des réactions alentour que de la formule elle-même, partagées entre la moquerie manifeste de l'opposition de droite et la posture anti-liberticide des partisans de gauche. L'ancien ministre de la Culture Jack Lang mit du sien en faveur de cette dernière option: refuser la notion de "bravitude", ce serait ignorer selon lui l'interprétation cachée du discours de Royal (la bravitude signifierait la plénitude du sentiment de bravoure, dit-il) et interdire à la langue française de jouer sur les variations sémantiques. Certes, tout mot trouve son sens dans l'usage de ceux qui l'emploient. Mais de là à suggérer une liberté totale dans l'usage et l'attribution de sens aux mots de la langue, il y a un pas de géant à accomplir et que les adeptes les plus conciliateurs de la langue de Molière ne souffriraient évidemment pas.

N'en va-t-il pas de même pour la "négritude", par ailleurs? C'est en substance ce que l'ancien Premier Ministre français Edouard Balladur avança pour ne pas s'associer à la moquerie générale de son groupe parlementaire: «Je ne comprends pas qu'on l'ait tellement critiquée. Je vous rappelle que Léopold Senghor (...) avait inventé la négritude. Et tout le monde a trouvé ça très bien ${ }^{1}$. » Non seulement le fameux concept des Etudiants Noirs rime pour beaucoup avec son prédécesseur, au destin plus malheureux ; mais de plus, son ambiguïté est telle que la notion de négritude fit couler beaucoup d'encre parmi les premiers défenseurs de la cause noire. Quel peut être l'effet attendu de cette expression devenue célèbre, à mi-chemin entre le pied de nez vengeur et l'essentialisme assumé ou maladroit?

Suivant la terminologie empruntée à la théorie des actes de discours, nous procéderons dans ce qui suit à une triple lecture de la «pensée noire ». La première lecture s'intéressera à l'aspect locutoire de ce discours, c'est-à-dire aux faits et références qui lui ont apporté son inspiration. La deuxième lecture portera sur l'aspect illocutoire, qui concerne l'intention de communication de l'émetteur et permettra de s'interroger sur l'objectif des écrivains de la négritude. La troisième et dernière lecture sera consacrée enfin à l'aspect perlocutoire qui rassemble l'ensemble des effets produits par le discours du locuteur/émetteur sur le comportement des interlocuteurs/récepteurs. Lecture plutôt qu'explication, au sens où la négritude ressemble à l'histoire mouvementée d'un bateau ivre dont le sens de la navigation est complexe sinon déroutant. Lecture plutôt que compréhension, dans la mesure où la proposition d'un ou plusieurs sens associés à la littérature noire n'empêche pas l'humilité du proposant et recommande de ne pas prétendre à la vérité unilatérale. Peut-on réellement comprendre la cause noire sans ressentir ou supporter son lourd héritage?

Entre interprétation et distorsion, les auteurs (blancs) de ces lignes ne doivent apporter leur propre écho que dans le cadre de la dernière partie du travail consacrée à l'aspect perlocutoire de la littérature noire. A moins que la couleur de départ ne soit qu'un prétexte historique au discours de révolte universel, sans couleur et susceptible d'être produit par toute personne victime de sa minorité? C'est cette ultime lecture que certains apôtres de la négritude ont revendiquée, parmi lesquels Frantz Fanon et Edouard Glissant. Nous conclurons par cette lecture décolorée ${ }^{2}$ de la

1 Voir l'article du 10 janvier 2007, dans Le Nouvel Observateur (Politique): "Bravitude: Balladur prend la défense de Royal".

2 Notre lecture "décolorée" signifie que la leçon de la négritude vaut pour toute sorte de révolte sociale. 
négritude, après en avoir examiné des versions plus sulfureuses que le politiquement correct cherche à effacer des mémoires publiques.

\section{Dialogue I : Les discours de la révolte (aspect locutoire)}

La négritude prend sa source dans l'océan, Atlantique. C'est dans cette immensité anonyme que le poète saint-lucien Derek Walcott (1930-) situe le mémorial à ciel ouvert de la civilisation noire et réalise son travail de deuil collectif:

Où sont vos monuments, vos batailles, vos martyrs?

Où est votre mémoire tribale?

Messieurs, dans ce gris coffre-fort. La mer.

La mer les a enfermés.

La mer est l'Histoire.

C'est surtout de la traite et de la mise en esclavage systématisée de la population noire que le courant littéraire, politique et philosophique de la négritude s'est inspiré bien malgré lui.

Tout commence avec la déportation organisée de populations issues de l'Angola et de l'Afrique équatoriale. Si l'Europe et l'Afrique noire n'ont pas le monopole intégral du rôle de bourreaux et de victimes dans le processus de traite et d'esclavage, la découverte du Nouveau Monde en 1492 puis l'accélération spectaculaire de l'économie de plantation (sucre, tabac) et minière expliquent en majeure partie l'équation produite entre-temps entre homme blanc et esclavagisme. Deux autres marchés ont illustré le commerce d'exploitation: la traite orientale du Moyen-Orient (Yémen, Golfe Persique), et la traite dite "intra-africaine" du continent noir lui-même (Dahomey, Soudan, sultanat de Zanzibar). A titre comptable, l'Occident et ses puissantes nations (Amérique anglaise, Portugal, France, Pays-Bas) serait responsable de 11 millions de déportations "atlantiques" entre le $\mathrm{XV}^{\mathrm{e}}$ et le $\mathrm{XIX}^{\mathrm{e}}$ siècle; quant au marché arabe (puis chinois) et au marché "intérieur" de l'Afrique, leur passif s'évaluerait respectivement à 14 millions de déportations du VII ${ }^{\mathrm{e}}$ au $\mathrm{XIX}^{\mathrm{e}}$ siècle et jusqu'à 6 millions pour le seul XIX ${ }^{\mathrm{e}}$ siècle. La responsabilité majeure imputée à l'Occident est due en somme à son expansion très rapide et au ratio de morts qu'il a provoqué en seulement cinq siècles contre douze siècles de déportation pour l'Orient.

La traite atlantique renvoie à une exploitation économique synonyme de déportation et de déracinement. Déportation vers l'Amérique du sud et les Caraïbes, lorsque l'extinction brutale du commerce amérindien et l'attribution de droits humains au peuple indien (suite à la controverse de Valladolid, de 1550 et 1551) justifia la priorité au commerce noir en guise de marchandise de substitution. Qualifié de "bien meuble" et de "bête de somme" par l'article 44 du Code Noir de Colbert (1685), l'“animal noir" ne jouissait d'aucune âme ni aucun droit particulier à l'égard de son maître omnipotent. De là l'organisation légale d'un marché triangulaire entre Afrique, Europe et Amérique. Malgré l'implication essentielle des pouvoirs maritimes de l'Espagne de Charles Quint et du Portugal, c'est dans la France de Louis XIV et à la colonie britannique des Amériques que la négritude trouvera son champ d'inspiration principal. Sa préhistoire militaire et littéraire peut en attester.

Quelques oppositions à l'esclavage sont à relever parmi des intellectuels: Tomaso Mercado et Bartolome de las Casas au XVI ${ }^{\mathrm{e}}$ siècle, Montesquieu et l'Abbé Grégoire au XVIII ${ }^{\mathrm{e}}$ siècle. La principale caution anti-esclavagiste des Lumières peut être associée à L'Esprit des Lois de Montesquieu (1748), où l'auteur s'attaque en règle aux arguments favorables à l'esclavagisme. Dans « De l'esclavage des nègres » (livre XV, chapitre V), le philosophe des Lumières use d'ironie pour réduire par l'absurde une série d'arguments jugés de mauvaise foi. Economiques, d'abord: « Le sucre serait trop cher, si l'on ne faisait travailler la plante qui le produit par des esclaves ». Ethnocentristes, ensuite: «Une preuve que les nègres n'ont pas le sens commun, c'est qu'ils font 
plus cas d'un collier de verre que de l'or, qui, chez les nations policées, est d'une si grande conséquence. » Physiques, aussi et surtout:

Ceux dont il s'agit sont noirs depuis les pieds jusqu'à la tête; et ils ont le nez si écrasé qu'il presque impossible de les plaindre (...) Il est si naturel de penser que c'est la couleur qui constitue l'essence de l'humanité, que les peuples d'Asie, qui font les eunuques, privent toujours les noirs du rapport qu'ils ont avec nous d'une façon marquée. (...) On ne peut se mettre dans l'esprit que Dieu, qui est un être très sage, ait mis une âme, surtout une âme bonne, dans un corps tout noir.

A noter toutefois que d'autres arguments du même Montesquieu atténueront son discours précédent, parmi lesquels l'argument climatique de la paresse inhérente aux peuples des pays chauds ou l'argument culturel du faible génie des peuples noirs.

Un plaidoyer en faveur de l'esprit noir paraîtra par la suite dans l'ouvrage majeur de l'Abbé Grégoire: De la littérature des nègres, ou recherche sur leurs facultés intellectuelles, leurs qualités morales et leur littérature (1808). On observe ici une sorte de préfiguration de la négritude, qui ajoute à la défense des droits fondamentaux de l'homme noir une ébauche de son génie intellectuel. L'auteur insista sur l'argumentation ethnocentriste des hommes blancs: «L'opinion de l'infériorité des nègres n'est pas nouvelle. La prétendue supériorité des blancs n'a pour défenseurs que des blancs juges et parties, et dont on pourrait d'abord discuter la compétence, avant d'attaquer leur décision », puis compléta sa défense des droits naturels du noir par une réfutation en règle des arguments physiques.

Mais dans la majeure partie des productions intellectuelles, philosophie et pouvoir politique se sont le plus souvent conjugués pour justifier la traite noire sur fond de hiérarchie raciale. Aristote et Ibn Khaldoun, Hegel et Kant ont tous participé à ce discours racialiste, essentialiste, générique et hiérarchique. Aristote a affirmé la servitude naturelle de certaines populations: «Ceux qui sont aussi éloignés des hommes libres que le corps l'est de l'âme, ou la bête de l'homme (et sont ainsi faits ceux dont l'activité consiste à se servir de leurs corps, et dont c'est le meilleur parti qu'on puisse tirer), ceux-là sont par nature des esclaves » (La politique, livre I, chapitre V), et Sepúlveda se servira largement de l'autorité intellectuelle du philosophe grec pour justifier l'esclavage des Indiens. Le noir est plus directement visé chez Ibn Khaldoun, légitimant ainsi la traite orientale dans son Introduction à l'histoire universelle et ajoutant à l'esclavage naturel admis par Aristote l'idée de servitude volontaire: « Les seuls peuples à accepter l'esclavage sont les Nègres, en raison d'un degré inférieur d'humanité, leur place étant plus proche du stade animal ». Son génie propre est tout bonnement réfuté par Kant, dont la classification esthétique des espèces humaines repose sur la création artistique et réduit le noir au statut d'enfant inapte:

Les Nègres d'Afrique n'ont reçu de la nature aucun sentiment qui s'élève au-dessus de la niaiserie. M. Hume invite tout le monde à citer un exemple par lequel un Nègre aurait prouvé des talents, et il affirme ceci: parmi les centaines de millions de Noirs qui ont été chassés de leurs pays vers d'autres régions, bien que beaucoup d'entre eux aient été mis en liberté, on n'en pas pourrait trouver un seul qui, soit en art soit en science, soit dans une discipline célèbre, ait produit quelque chose de grand. Parmi les Blancs, au contraire, il est constant que certains s'élèvent de la plus basse populace et acquièrent une certaine considération dans le monde, grâce à l'excellence de leurs dons supérieurs. Si essentielle est la différence entre ces deux races humaines! Et elle semble aussi grande quant aux facultés de l'esprit que selon la couleur de la peau ${ }^{3}$.

La philosophie des Lumières n'a pas échappé non plus à ce discours sur les espèces, puisque Voltaire valida le système esclavagiste à son tour. Les tournants révolutionnaires de la fin du XVIII siècle produisirent une modification des consciences et du rapport à l'Autre dont se nourrira plus tard la philosophie subjective du $\mathrm{XX}^{\mathrm{e}}$ siècle. Mais il va de soi que la population asservie décida d'agir sans attendre les quelques réflexions de penseurs blancs favorables à leur cause.

3 Voir Kant: Observations sur le sentiment du beau et du sublime, section IV: " Des caractères nationaux, en tant qu'ils reposent sur le sentiment différencié du sublime et du beau. 
Avant les révolutions et leurs idées abstraites, la révolte des hommes de chair et d'os avait marqué par intermittence l'histoire du commerce maritime et ses déportations à fond de cale. Briser les chaînes et provoquer des mutineries fut à l'histoire de la mer coloniale ce que ces jacqueries paysannes fut à l'histoire des terres impériales: une révolte de corps et d'esprits décidés à défendre leur cause sinon perdue. Côté français, la Révolution de 1789 joua un rôle décisif dans la prise de conscience d'un peuple noir susceptible de défendre son droit à l'existence par le droit positif. Une Société des amis des Noirs avait déjà été établie en 1788 par quelques révolutionnaires émancipateurs (Brissot, Clavière, Mirabeau), quelles que soient les raisons profondes de cette philanthropie déclarée. Mais la véritable lutte noire trouve son expression dans l'usage des armes face au maitre ancestral.

Quelques dates prolongent dans l'Histoire du temps les multiples révoltes silencieuses, privées de chronologie. En 1791, l'île de Saint-Domingue (future Haïti) connut une insurrection de ses esclaves et causa la mort d'un millier d'exploiteurs blancs ou békés (créoles descendant des premiers colons européens). Cette révolte aboutit à l'abolition de l'esclavage: à Saint-Domingue (le 24 juin 1793), puis dans l'ensemble des colonies sur décision de la Convention (04 juin 1794). A la vague de libération progressive succéda l'intermède de Napoléon Bonaparte, synonyme de rétablissement de l'esclavage légal dans le nouvel Empire français. En 1801, l'abolitionniste guadeloupéen Louis Delgrès prit la tête d'une rébellion face aux troupes napoléoniennes avant que le célèbre Toussaint Louverture ne proclame une nouvelle constitution des hommes noirs à Saint-Domingue. Malgré la répression générale des révoltes caribéennes, la victoire du général Jean-Jacques Dessaline conduisit à l'indépendance de la nouvelle Haïti en 1804 mais n'avait pas empêché pour autant l'esclavagisme d'être rétabli sur les territoires français d'outre-mer dès 1802. Pas plus que dans l'Empire du Royaume-Uni voisin et les Etats-Unis libérés du joug britannique, où la traite atlantique fut abolie en 1807 mais ne signifiait pas la fin de l'esclavage dans les colonies américaines. Tout au contraire: si la fin totale de la traite et du commerce atlantique ne fut officialisée que lors du congrès de Vienne de 1815, elle serait loin de signifier la fin de l'esclavage en tant que tel et devrait conduire bien plutôt à la colonisation du continent africain par les pouvoirs occidentaux. Le Congrès de Berlin de 1884 finalisera le jeu systématique de cette installation à visage plus humain, planifiée sous les auspices du chancelier Bismarck et teintée d'une mission civilisatrice. De même pour les Etats d'Unis d'Amérique, où la progression des droits physiques du noir s'accompagne de lois ségregationnistes en 1896: les hommes noirs et blancs sont déclarés « égaux, mais séparés ». Le pouvoir politique reste dans les mains du blanc, ce qui ne sera pas sans incidence dans le rapport de force entre consciences dominantes et dominées.

D'autres hauts faits militaires ont honoré la cause noire avant cela, parmi lesquels deux notoires: la bataille d'Ishandhlwana en 1879, ou la victoire magistrale de l'armée zouloue sur l'armée anglaise en Afrique du Sud; la bataille d'Adoua en 1869, ou la victoire du Négus Ménélik II d'Ethiopie sur l'Italie en 1896. Ce dernier événement inspirera aussi bien le panafricanisme et l'éthiopianisme de Joseph Casely Hayford et du prophétique Marcus Garvey que le rastafarisme de Bob Marley. D'autres victoires politiques ont suivi les premiers succès militaires de la cause noire, bien avant la libération du leader de l'ANC sud-africain Nelson Mandela en 1990 ou l'élection du premier président noir des Etats-Unis Barack Obama en 2008. Citons notamment l'indépendance du Libéria en 1847, premier Etat d'Afrique déclaré après avoir servi de terre de rapatriement dès 1822 pour les esclaves caribéens affranchis. La seconde révolution républicaine française de 1848 consacra aussi le nom de Victor Schoelcher, auteur le 27 avril d'un décret d'abolition de l'esclavage signé par le ministère des Affaires Etrangères Lamartine.

La négritude francophone trouve sa source d'inspiration dans les lieux de traite et de colonisation: le discours du déracinement dans les Antilles françaises (Guadeloupe, Haïti, Martinique); le discours de la colonisation dans l'Afrique occidentale (l'AOF formée en 1895) et équatoriale (l'AEF de 1919). Quant à la diaspora noire de l'Amérique anglophone, elle s'inspirera elle aussi de la négritude francophone et de sa problématique basée sur les questions d'identité et 
d'altération forcée. La dernière période marquante de décolonisation et d'émergence consécutive du tiers-mondisme ouvrira définitivement les vannes de la littérature noire, produisant une réflexion en retour sur les phases de libération individuelle et collective. Comment garantir une libération totale et compléter la victoire psychologique des consciences noires par une victoire politique des nations noires? Après le discours de De Gaulle à Brazzaville en 1944, la phase d'émancipation politique conduira à l'indépendance de dix-huit pays africains en 1960, puis à la création plus tardive de l'Organisation de l'Unité Africaine en 1963 et à l'Union Africaine en 2002. D'autres événements ont servi de repères collectifs pour le peuple noir américain: l'affaire Rosa Parks de 1955, où le refus de céder sa place de bus à un homme blanc lancera le mouvement civique aux Etats-Unis et aboutira au rêve (1963) puis à l'assassinat (1968) de Martin Luther King. D'autres organisations et luttes intestines auront retenu l'attention d'une histoire toujours moins minuscule, entre-temps: Black Panther Party for Self Defense et Nation of Islam, héritiers du panafricanisme de Marcus Garvey et son idéal de retour à la terre ancestrale comparable au sionisme juif ${ }^{4}$; un tournant dans l'histoire juridique du peuple afro-américain est produit par le Civil Rights Act du président Lindon Johnson, qui interdit toute discrimination raciale dans le pays. Cette progression en droit n'empêchera pas la condition noire de stagner en fait, témoins les émeutes à distance du quartier Watts de Los Angeles (1965, puis 1992).

L'ultime étape du rapport entre dominants et dominés opère une sorte de renversement moral et juridique à la fois. Notamment en France, qui rime de nos jours avec repentance: en 2001, la loi Taubira fait du colonialisme français un crime contre l'humanité, tandis que la loi Gayssot sanctionne d'amende ou de prison toute incitation à la haine raciale depuis 1990. Mais la loi ne change pas les esprits aussi facilement: la création de SOS Racisme en 1984 n'a pas éradiqué ce que William Edward Burghardt Du Bois qualifiait dès 1900 de problème du XXe siècle, à savoir: celui de la démarcation fondamentale entre blancs et noirs. Au discours cosmopolite et promoteur de métissage a succédé parfois une résurgence du séparatisme et du nationalisme noir: la Tribu Ka de Kémi Seba a beau avoir été dissoute sous le gouvernement de Villepin, elle n'en témoigne pas moins d'une ambiguïté profonde dans le discours de l'émancipation noire: intégration, ou séparation à l'égard de la population blanche? Réconciliation ou indifférence, voire vengeance pour solde de tout compte? Au racisme blanc succède par endroits un racisme à rebours, celui du noir impossible à consoler et que l'héritage sanglant empêche de pardonner à titre de fidélité. Il en va de même sur le continent noir, où la multiplication des guerres civiles qui suivit la fin de la guerre froide manifeste en partie un retour à de vieux contentieux issus de la traite intra-africaine. Ne réduisons cependant pas la tendance actuelle du séparatisme à une affaire purement interne: les oppositions entre populations africaines doit autant aux blessures mémorielles qu'à ce que les "négrologues" incriminent sous l'appellation d'esprit tribal ou singulier. C'est précisément contre ce repli identitaire que la négritude a proclamé son mot d'ordre, malgré un discours vacillant parfois entre essentialisme contre-productif et littérature stérile (dixit le verdict sévère du philosophe béninois Stanislas Spero Adotevi). Qu'elle soit d'ordre inter-racial (noirs contre blancs), intra-racial (populations noires) ou trans-racial (catégories sociales, alliances stratégiques avec le partenaire puissant de la Françafrique), la déchirure africaine constatée au cours des vingt dernières années (Rwanda, Congo, République centrafricaine, Côte d'Ivoire, Mali, Erythrée, Soudan, Somalie, etc.) constitue un défi d'actualité pour le courant de la négritude et son discours post-racial. Revenons sur ses principaux auteurs, tout en y associant l'intelligentsia américaine et ses procédés similaires.

La négritude est un jeu d'esprits à somme non-nulle; rien de ludique dans cette partie où l'auteur doit conquérir le lecteur et renverser le cours d'une Histoire assimilant la noirceur au Mal. A commencer par l'héritage culturel du peuple noir tout entier, que les monothéismes juif et chrétien

4 En témoignent ces paroles issues de la tradition du chant des negro spirituals: « Le Seigneur a dit à Moïse ce qu'il faut faire - Laisse partir mon peuple - Ramener les enfants d'Israël - Laisse partir mon peuple» (in «Prends ma main, doux Seigneur, montre-moi le chemin du retour »). 
ont condamné dans le texte. La damnation de Cham est à la négritude ce que la malédiction d'Eve peut être au féminisme: un mythe originaire auquel doit répondre un combat rhétorique, une réparation par le texte. Cham, fils de Noé, est l'ancêtre biblique du Noir dans le livre de la Genèse:

Noé commença à cultiver la terre, et planta de la vigne. Il but du vin, s'enivra, et se découvrit au milieu de sa tente. Cham, père de Canaan, vit la nudité de son père, et il le rapporta dehors à ses deux frères. Alors Sem et Japhet prirent le manteau, le mirent sur leurs épaules, marchèrent à reculons, et couvrirent la nudité de leur père; comme leur visage était détourné, ils ne virent point la nudité de leur père. Lorsque Noé se réveilla de son vin, il apprit ce qui lui avait fait son fils cadet. Et il dit: Maudit soit Canaan! Qu'il soit l'esclave des esclaves de ses frères! Il dit encore: Béni soit l'Eternel, Dieu de Sem et que Canaan soit leur esclave! Que Dieu étende les possessions de Japhet, qu'il habite dans les tentes de Sem, et que Canaan soit leur esclave ${ }^{5}$ !

La réplique fut donnée entre autres par un métis: Frederick Douglass (1818-1895), porte-parole de la communauté noire américaine et convaincu que la propagande blanche devait trouver objecteur sans heurt ni violence. Dans ses Mémoires d'un esclave, il analyse du système esclavagiste vaudra un premier contre-argument à la malédiction religieuse :

Si, en vertu du Livre, seuls les descendants directs de Cham peuvent être tenus en esclavage, alors, pour la même raison, l'esclavagisme du Sud ne pourra bientôt plus être légitime; chaque année, des milliers d'esclaves y sont en effet mis au monde qui, tout comme moi, doivent la vie à des pères blancs qui sont aussi le plus souvent leurs maîtres. ${ }^{6}$

Le discours s'endurcit avec le sociologue William Edward Burghardt Du Bois (1868-1963), un des fondateurs du NAACP (National Association for the Advancement of Colored People, 1909) opposé à la posture trop prudente de l'intégrationniste par le travail Booker T. Washington ${ }^{7}$. Jusqu'à déboucher sur le courant radical du panafricanisme, et son vœu de retour à la terre première (à défaut d'être promise). Lors du Premier Congrès Panafricain de Londres, en 1900, Du Bois lança sa fameuse formulation du "problème noir" et proposa une solution séparatiste comparable aux tendances de l'époque: pangermanisme, panhéllenisme, mais aussi et surtout le sionisme et son appel au retour. Loin des clichés pacifistes du métissage harmonieux, le Jamaïcain Marcus Garvey (1887-1940) prit fait et cause pour le courant religieux et mystique de l'éthiopianisme; pas d'intégration cosmopolite, mais la séparation volontaire en direction du continent d'origine. Les paroles qui suivent vaudront à Garvey de passer pour prophète en la matière, préfigurant l'avènement du ras Tafari Mekonnen dit Haïlé Sélassié (couronné Empereur d'Ethiopie en 1930) :

Je veux que vous, hommes et femmes de couleur à Newport News, réalisiez que Dieu vous a créés pour un dessein; ce dessein vous devez le vivre. Dieu a dit, à travers l'auteur des Psaumes, que l'Ethiopie doit tendre les mains vers lui et que des princes sortiront de l'Ethiopie. Je crois avec ferveur que l'heure est venue pour l'Ethiopie de tendre ses mains vers Dieu, et que c'est précisément ce que nous faisons à New York, en Pennsylvanie, dans les Indes occidentales, en Amérique centrale, en Afrique et partout dans le monde ${ }^{8}$.

5 Genèse, chapitre 9, 20-27. Aucune référence à la couleur ne justifie ici la malédiction du peuple noir; l'association est due plutôt à la filiation de Cham, père de Cusch (signifie "noir" en hébreu ancien et désigne la Nubie, ou actuel Soudan), ainsi qu'à ces propos du Talmud sur l'apparence physique de la descendance de Cham: «Il faut donc que ce soit Canaan, ton premier né, qu'ils prennent pour esclave. Et comme tu m'as rendu incapable de faire de vilaines choses au plus noir de la nuit, les enfants de Canaan naîtront vilains et noirs! De plus, puisque tu t'es contorsionné pour voir ma nudité, les cheveux de tes petits enfants s'entortilleront jusqu'à devenir crépus et ils auront les yeux rouges, en outre, puisque tes lèvres ont plaisanté sur mon infortune, les tiennes vont enfler et puisque tu as manqué d'égards pour ma nudité, ils iront tous nus et leur membre viril s'allongera ignominieusement. »

6 F. Douglass, 2007, p. 5-6.

7 Ce souci d'intégration s'accompagne d'un souci de bonne représentation: celle du "Bon Noir" coopératif (les Oncles Tom et Ben's, ou $Y^{\prime}$ a Bon Banania), par opposition au portrait inverse du noir maléfique ou simplement stupide (le personnage Jim Crow). On le retrouve dans les mass médias modernes, à travers des personnages inoffensifs de séries américaines tels que Bill Cosby ou Steve Urkle et Carl Winslow, bon patriarche policier et papa poule embourgeoisé (dans « Family Matters »).

8 «Le Nouveau Nègre et l’Unia », discours du 25 octobre 1919. Unia: Universal Negro Improvement Association and 
Le panafricanisme de Garvey est associé également à ce qui sera qualifié par la suite de nationalisme noir, expliquant ainsi son admiration pour un Mussolini qui n'en envahira pas moins la terre sacrée d'Abyssinie. Du pangermanisme au panafricanisme déjà mentionnés, le mot d'ordre est identique: un peuple, une nation. Avec le souci de construire un régime à taille continentale, basé sur la concorde et le socle commun de la déportation. Un mot d'ordre qui a de quoi surprendre, par rétrospection:

Depuis cinq ans l'Association Universelle pour le progrès nègre a défendu la cause de "l'Afrique aux Africains" - à savoir l'idée que dans le monde entier les peuples nègres devraient se concentrer sur un objectif: se construire pour euxmêmes une grande nation en Afrique. (...) Il ne s'agira pas d'aller en Afrique dans le but d'exercer un pouvoir féodal sur les indigènes, ce que vise l'Unia en Afrique, c'est une coopération fraternelle où les intérêts des Africains et des Nègres américains et antillais se confondront; nous devons entrer dans une communauté de partenaires pour bâtir l'Afrique selon les intérêts de notre race. (...) Le Nègre a suffisamment pâti de cette pratique tant prisée de la supériorité raciale qui lui fut infligée par d'autres, pour ne point tolérer semblable présomption de la part des siens 9 .

Cela étant, la tragédie collective chevillée au corps de l'homme noir le met à distance d'un quelconque idéal de pureté de la race. La promiscuité entre l'esclave et son maître donna lieu à de multiples métissages et ses palettes de couleurs hiérarchiques: békés puis mulâtres, chabins puis coulis profitent d'un moindre affranchissement, puis un certain accès à l'écriture tant que le servant sert le maître et admet sa supériorité. Premier acte de métissage culturel, également: le créole constitue la langue du maître que l'esclave s'approprie dans la parole du conteur (en référence au griot ou communicateur traditionnel africain) et reproduit par le biais des écrits mulâtres (à moitié blancs, donc affranchis). On retrouve dans cette pratique de 1" oraliture" le rôle central que le griot tient en Afrique dans la transmission orale des héritages communs: contes, proverbes, chants traditionnels et luttes de tambouyés sont maintenus sur les lointaines terres des Caraïbes pour préserver le souvenir essentiel des terres perdues.

Après la réfutation des sentences blanches, la négritude ne peut émerger encore sans une reconstruction complète de la culture noire. On parle ainsi d'un "nouvel humanisme d'inspiration africaine" qui, à l'inverse des séparatistes noirs, promeut la mixité raciale mais pas sans annuler tout d'abord le rapport de force défavorable entre l'élite blanche et la main d'œuvre noire. Dans cet ordre d'idées, le néopharaonisme égyptien de l'historien américain Carter G. Woodson (1875-1950) compte rendre aux siens leur passé illustre et affirmer l'identité noire de la civilisation égyptienne ${ }^{10}$. La littérature afro-américaine de l'entre-deux-guerres se veut plus légère, moins axée sur un passé à reconquérir et plus soucieuse de jouir du présent. L'écrivain Langston Hughes (1902-1967) répond à la ségrégation d'Etat par l'ironie mordante: il raille le mot d'ordre "just be simple" adressé à la population noire soumise et en tire ses Simple Stories, tout en se détournant d'un afrocentrisme trop théorique à son goût. Face à la difficulté du quotidien noir, Hughes croit plus modestement au rôle de la musique et des arts en vue d'un changement général des mentalités. De même pour Claude McKay (1889-1948), persuadé quant à lui du pouvoir de la musique sur les consciences et préfigurant par l'exemple la future "raison-étreinte" fusionnelle de Senghor. Dans Banjo, McKay exhorte à remuer tout ça (« Shake that thing ») par la danse et la musique des cafés bigarrés:

Il y avait là des Noirs de toutes les nuances de peau. Les mulâtres eux-mêmes avaient daigné descendre de leur piédestal pour se mêler à cette foule. Car, pas plus qu'aux Antilles britanniques et qu'en Afrique du Sud, les mulâtres des

African Communities League.

9 «L'Afrique aux Africains », 1920; italiques des auteurs.

10 Mitstraïm, fils de Cham, porte un nom sémitique qui signifie "Egypte"; d'après l'anthropologue et politicien sénégalais Cheikh Anta Diop (1923-1986), la civilisation européenne de Champollion a "blanchi" cette Egypte pour priver l'Afrique de son illustre passé et la maintenir dans son image de sous-civilisation mineure: «La naissance de l'égyptologie sera donc caractérisée par la nécessité de détruire à tout prix et dans tous les esprits, le souvenir d'une Egypte nègre, de la façon la plus complète. » (« Nations nègres et culture », in Présence Africaine, 1954) 
colonies françaises ne se mélangent ordinairement avec les Nègres. Mais un magicien les avait tous rassemblés pour "secouer ça" et lamper du vin rouge, du vin blanc, du vin doux. Et aussi les Noirs d'Afrique occidentale anglaise, les Noirs du Portugal et d'Amérique, tous ceux que leur dérive avait amenés dans ce port où passe le monde entier.

De façon générale, la danse a pour fonction de réhabiliter les vertus d'un corps autrefois meurtri et capable de produire cette fusion interraciale que décrivent Hughes et McKay. On parlera plus tard d'un roman réaliste négro-américain, loin des recherches anthropologiques et plus proche de l'homme quotidien.

Impossible enfin d'évoquer la culture noire sans ajouter à la littérature le rôle de la musique. Ce qui servirait d'argument moqueur pour le blanc raciste n'est pas renié par l'artiste engagé noir: non pas que ses frères d'infortune aient le "rythme dans le sang", conformément à une logique génétique dont la population noire a largement payé les frais. Le rythme se situe davantage dans la mémoire, qui se veut collective et propice à la transmission orale. Impossible donc de ne pas mentionner la longue génération de musiciens et styles musicaux inspirés par l'économie de plantation, d'abord (gospel, blues), puis par l'urbanité de seconde zone ensuite (funk, hip hop, rap, reggae). Entre chants sacrés et industrie du loisir, la musique noire s'inscrit aussi dans la lignée du mouvement pour les droits civiques et conjugue tour à tour dignité individuelle, panafricanisme et revendication sociale. Martin Luther King fut assassiné l'année où James Brown déclara «Say it loud: I am black and proud », tandis que la génération suivante du hip hop jonglera entre diatribe radicale et récupération commerciale. Bob Marley chanta quant à lui les discours de la fierté, de l'unité et du panafricanisme que Du Bois et Garvey avaient argumentés plus tôt. La musique apparaît comme un instrument de masse accessible et efficace, une autre façon de poursuivre la lutte des esprits par le biais de flows, où maîtres de cérémonies et disc jockeys reprennent à leur façon le flambeau d'un combat idéologique à poursuivre. Par le cri de révolte populaire, on prête à la musique la vertu d'une éducation de masse underground capable d'affranchir la minorité noire de l'idée d'esclavage de masse ou d'aliénation en tous genres. Le graffiti est l'autre forme d'expression de la cause noire contemporaine, partant du principe que le trafic d'influence publique dont l'égalité des droits sociaux est l'enjeu se propage plus vite par la persuasion rapide des images que par la conviction patiente des arguments.

La parole politique, enfin, apporte à la culture noire son principal contenu locutoire pendant les années 60. Cette décennie marque l'émergence du Black Power et du principe d'autodéfense noire en général. Le Black Panther Party est fondé en 1966 par Bobby Seale et Huey Newton, où se manifeste l'inspiration double du tribun américain Malcolm X et de l'écrivain martiniquais Frantz Fanon. Rhétorique et sophistique se disputent la faveur de l'opinion publique: lorsque des gouverneurs d'Etat assimilent la lutte pour les droits civiques des noirs à des provocations marxistes en pleine période de guerre froide, les champions olympiques du 200 mètres Tommie Smith et John Carlos lèvent le poing ganté sur le podium de Mexico en 1968. A la violence des mots s'allie celle des actes, même si le principe d'autodéfense communautaire est présenté comme une simple condition préalable à l'intégration civique. Stokely Carmichael et Charles V. Hamilton exposent la méthode et l'objectif du mouvement sous les traits d'une armée civique d'avant-garde : « Le Black Power repose sur un postulat fondamental: avant d'entrer dans la société ouverte, un groupe doit commencer par serrer les rangs ». ${ }^{11}$ Ils se défendent de procéder à un racisme inversé, et se justifient par un souci de reconnaissance plutôt que de domination:

Il n'y a aucune analogie - quelque effort d'imagination que l'on puisse faire - entre les avocats du Black Power et les racistes blancs. Le racisme, ce n'est pas seulement exclure un autre pour des raisons raciales, c'est l'exclure pour le dominer ou continuer à le dominer. (...) Ceux qui veulent l'autodétermination, le droit d'être identifiés, donc les partisans du Black Power, désirent participer pleinement aux prises de décisions qui affectent la vie des Noirs et exigent que leurs vertus en tant que peuple soient reconnues. ${ }^{12}$

11 M. Carmichael \& C. V. Hamilton, 1968, p. 82.

12 Ibid., p. 85. 
Quant à la non-violence prônée par le pasteur Martin Luther King, les sympathisants du rival Malcolm X n'y voient qu'une perte de temps et lui préfèrent la démonstration de force pour conjurer le sort pluriséculaire: «Les partisans du Black Power ne veulent plus de slogans et de l'éloquence creuse propres à la lutte pour les droits civiques de ces dernières années. Progrès, nonviolence, intégration, crainte du "recul blanc", coalition: ce langage appartient au passé, il est maintenant vide de sens ${ }^{13}$. »

Une autre allégation nuira bien plus à la cause noire: celle d'antisémitisme. Dans le cadre des luttes d'influence médiatique, celles que les représentants afro-américains doivent apprendre à contrôler pour parvenir à leur fin (l'égalité des droits sociaux), l'idée de rejeter le juif pour ce qu'il est ne peut que servir le pouvoir en place s'il reproduit la logique blanche du racisme et entre en contradiction flagrante avec les intentions déclarées du mouvement noir pour les droits civiques. La réponse de la Nation of Islam sera constante: la critique n'est pas d'ordre ontologique, mais historique; elle ne vise pas le Juif en soi, mais des Juifs situés. Le mot d'ordre se transmet jusque dans les termes du plus récent et controversé Louis Farrakhan, qui associe par ailleurs le paradis blanc à l'enfer noir et parle d'êtres sataniques opposés à la bonté des êtres solaires ${ }^{14}$. Farrakhan mobilise l'Histoire ancienne pour expliquer le contentieux présent avec les juifs:

Où Donc, quand les Juifs furent châtiés, battus et assassinés, ils quittèrent l'Espagne. Où êtes-vous allés? Aux Caraïbes. En Amérique du Sud et vous êtes devenus des propriétaires de plantations ... C'est pour cette raison que beaucoup de Juifs ne veulent pas entendre parler de réparations. Je ne suis pas habité par la haine, enlevez-vous immédiatement cela de l'esprit. Mais j'ai la passion de la vérité et de la justice ${ }^{15}$.

Face à ce contenu aussi riche que pesant, la négritude francophone a trouvé sa propre voie sans renier aucune des manifestations de la révolte noire. Comment concilier cet ensemble pétri de contradictions, et dans quelle mesure la négritude peut-elle apporter un projet de civilisation viable? Après une description du contenu locutoire, et non sans avoir déjà exprimé quelques intentions des personnages, examinons à présent ce que nous avons proposé d'appeler l'aspect illocutoire de la négritude: ses intentions fondamentales, par-delà les événements historiques qui ont pu l'inspirer.

\section{Dialogue II : Les identités de la raison (aspect illocutoire)}

La contradiction est au cœur de la pensée noire, comme peuvent l'être les réactions d'un homme

\section{Ibid., p. 87.}

Autre témoignage d'une ségrégation, celui de Malcolm X: «Monsieur Muhammad nous enseigne que l'intégration n'est qu'une astuce des Blancs à l'heure actuelle pour endormir les Noirs, pour les endormir en leur faisant croire que le Blanc change, et qu'il veut réellement que nous restions ici; mais l'Amérique elle-même, à cause du grain qu'elle a semé dans le passé contre le Noir, est bien près de récolter la tempête aujourd'hui, de récolter ce qu'elle a semé ... l'Amérique doit payer aujourd'hui le crime qu'elle a commis en tenant les Nègres en esclavage. » (Nous, les Nègres, James Baldwin, Martin Luther King, Malcolm X: Entretiens avec Kenneth B. Clark, trad. André Chassigneux, La Découverte, 2008)

14 "White Man's Heaven is a Black Man's Hell", (déclaration lors de la Million Man March de Washington (octobre 1995); à quoi il ajouta les propos de Leonard Jeffries (1937-) sur la nature du blanc: un "diable aux yeux bleus" issu des peuples cruels du froid ("ice people") et opposé au peuple noir chaleureux ("sun people").

15 « Saviour's day speech », discours prononcé à Chicago, le 29 février 2004.

Malcolm X s'associait aux paroles de Muhammad: «Les partisans de Monsieur Muhammad ne sont anti-rien, sauf anti-injustice, anti-exploitation et anti-oppression. Beaucoup de Juifs ont un complexe de culpabilité quand on parle d'exploitation, parce qu'ils se rendent compte qu'ils contrôlent $90 \%$ des affaires dans toutes les communautés noires de l'Atlantique au Pacifique, et qu'ils tirent plus de bénéfices du pouvoir d'achat des Noirs que les Noirs euxmêmes, ou toute autre fraction de la communauté blanche. " (Nous, les Nègres, James Baldwin, Martin Luther King, Malcolm X: Entretiens avec Kenneth B. Clark, trad. André Chassigneux, La Découverte, 2008); cette phase de rejet de l'autre prendra une autre tournure suite à son pèlerinage de 1964. 
blessé. L'opposition contradictoire entre les programmes d'intégration et de séparation du peuple noir a été mise en évidence en ces termes bibliques:

Le leadership afro-américain a toujours eu tendance à être divisé en couples d'opposés: il y a eu un Esaü pour chaque Jacob. L'élégant ex-esclave Frederick Douglass faisant face au militant nationaliste Henry H. Garnet, le radical W. E. B. Du Bois au représentant du courant majoritaire Booker T. Washington, et l'intégrationniste Martin Luther King au séparatiste Malcolm $\mathrm{X}^{16}$.

Apparu pour la première fois en 1934 dans la revue L'Etudiant Noir ${ }^{17}$, le concept de négritude constitue une réflexion sur les identités de la raison. Il faut entendre ce projet de deux façons distinctes, selon que l'on insiste sur le premier terme d'identité ou le deuxième terme de raison: non seulement comme une étude des identités fondées sur plusieurs raisons, que ce soit le rejet de l'autre ou la reconnaissance de soi; mais aussi comme une étude de l'identité des types de raison à l'œuvre chez le noir et le blanc, qu'elle soit intuitive chez le premier ou discursive chez le second. Essayons de décrire ces nuances afin de saisir l'objectif, l'enjeu et la problématique de la négritude.

Assimilation et séparation ont servi de points cardinaux pour la cause noire. Booker $\mathrm{T}$. Washington et Martin Luther King, dans le premier cas; Marcus Garvey, Malcolm X et le nationalisme noir en général, dans le second cas. Le racisme inversé dont ces derniers ont souvent été taxés pourrait résulter d'une méprise générale, relative à leur logique d'une fortification identitaire en plusieurs étapes et passant par l'affirmation première de soi. Le premier stade de la fierté retrouvée se retrouve dans les textes de l'écrivain Jean-Price Mars, défenseur du nationalisme haïtien. Dans Ainsi parla l'oncle, le processus de réhabilitation passe ainsi par une prise initiale de conscience du "retard africain", dû à l'esclavage mental et raconté par une fable mythique:

Pourquoi, nous Haïtiens, nous sommes encore arriérés dans la course du progrès? La légende racontera que certain jour, Dieu ayant achevé l'œuvre de la création, manda par-devant son trône le Blanc, le Mulâtre et le Nègre, et leur tint à peu près le langage suivant: - Voici, je veux doter chacun de vous d'aptitudes spéciales. Exprimez vos désirs, je les agréerai aussitôt. Le "Blanc" incontinent, sollicita la domination du monde par la sagesse, la fortune, les arts et la science. Le Mulâtre désira ressembler au Blanc - ce qui était d'ailleurs se mettre un peu à sa suite - mais quand vint le tour du Nègre, le récit atteint le plus haut burlesque. -Et vous mon ami, fit le bon Dieu, que désirez-vous ? Le Nègre s'intimida, bredouilla quelque chose d'inintelligible, et, comme le bon Dieu insistait, le Nègre pirouetta et finit par dire: "M'pas besoin angnin. Cé ac ces Messié là m’vini”" (Je n'ai besoin de rien. Je suis le serviteur de ces Messieurs). Et voilà pourquoi nous sommes encore à la suite $(\ldots)^{18}$.

En pleine période de décolonisation et de réaction contre l'impérialisme, la lutte forcée des races a souvent rejoint la lutte assumée des classes au sein de la communauté noire, jusque dans les écrivains de la négritude: Césaire fut d'abord membre du PCF, tout comme furent sympathisants du communisme à l'étranger des penseurs tels que les Trinidadiens George Padmore (1903-1959) et Cyril Lionel Robert James (1901-1989) ou le Kenyan Jomo Kenyatta (1894-1978). Elle resta sinon un règlement de compte en interne entre l'homme noir et son passé riche de sens. L'affirmation de soi prend alors la forme d'un rejet catégorique de l'autre: chez le bien nommé Malcolm X et son maître à penser Elijah Muhammad de la Nation of Islam, où le projet initial d'un Etat noir dans le sud des Etats-Unis devait préparer à un retour définitif en Afrique et répondre à la "farce de Washington" du 28 août 1963 par un rejet de principe du blanc raciste. Indifférence face à la mort d'un Kennedy trop blanc pour être honnête; antisémitisme économique, sur des arguments extraits de la traite atlantique et complétés par une accusation d'exploitation des ghettos afro-américains.

Dans une acception plus politique, l'affirmation de soi vaut aussi à l'échelle des Etats et s'applique au cas du panafricanisme: l'unité du continent africain est présentée comme la condition

16 Voir Henri Louis Gates Jr.: Thirteen Ways of Looking at a Black Man, 1997.

17 Trois noms sont associés à cette publication: le Martiniquais Aimé Césaire (1913-2008), le Sénégalais Léopold Sédar Senghor (1906-2001), et le Guyanais Léon-Gontran Damas (1912-1978).

18 J. PRICE-MARS, 2009, p. 63. 
nécessaire à l'existence de l'Afrique sur la scène internationale. Ainsi l'explique le futur président du Ghana Kwame Nkrumah (1909-1972), promoteur des Etats-Unis d'Afrique avant le sommet des trente chefs d'Etat de l'Afrique indépendante (22-23 mai 1963): « Nous devons tous tirer une leçon de l'Europe, par contraste. Cultivant par trop ses nationalismes exclusifs, elle a sombré, après des siècles de guerres entrelardées d'intervalles de paix instable, dans un état de confusion, simplement parce qu'elle n'est pas parvenue à se donner une saine base d'association politique et de compréhension (...) Tant que nous, les Africains, pour qui l'unité est le but suprême, nous efforçons de concerter nos efforts dans ce sens, les néocolonialistes font tout pour les rendre vains en encourageant la formation de communautés fondées sur la langue des anciens colonisateurs. » (L'Afrique doit s'unir, Payot, 1964, traduction Laurent Jospin, Présence Africaine, 1994 Payot, 1964). La réalité politique liquidera l'espoir déçu de Nkrumah, quand on sait que la création de l'Organisation de l'Unité Africaine en 1963 n'aboutira qu'à une Afrique des nations morcelée, déchirée par des ambitions séparées et mettant fin à l'ambition d'un système-empire unifié. Ce qui vaut pour les nations noires vaut également pour les individus noirs, d'où la mission périlleuse pour une négritude destinée à préparer une conscience noire.

Or si conscience noire il y a, de quoi est-elle nom ou à quel égard se met-elle en évidence? Quelques propriétés caractéristiques avaient été relevées parmi les sciences sociales des siècles derniers. Chez le diplomate et écrivain Joseph Arthur de Gobineau (1816-1882), d'une part. Bien que son nom soit couramment lié aux principaux théoriciens du racisme européen, la thèse de l'inégalité des races de Gobineau amène aussi à valoriser le noir comme un être imaginatif: «Le mélange avec l'espèce noire, lorsqu'il est léger, développe l'intelligence chez la race blanche, en tant qu'il la tourne vers l'imagination, la rend plus artiste, lui prête des ailes plus vastes » (Essai sur l'inégalité des races humaines, 1853). Chez le philosophe, sociologue et anthropologue Lucien Lévy-Bruhl, d'autre part: d'après lui, l'homme noir est fondamentalement lié à la nature et vit pour cela dans un «état émotionnel étrange pour nous mais naturel » (L'âme primitive, 1927). L'auteur parlera ensuite de "mentalités prélogiques" distinctes de l'homme occidental, tout en doutant cela dit de la pertinence scientifique de l'expression.

Il va presque de soi que l'inégalité des races ou la prélogicité ne sauraient être revendiquées par les apôtres de la négritude. Le projet de définition de l'homme noir prend une toute autre tournure chez eux, à en croire le projet cosmopolite qui suit: «Quand il dénonce avec véhémence la violence et le mensonge social, Senghor invite à la refondation de l'humanisme et de la civilisation de l'universel, qui n'est rendue possible que par le dialogue de toutes les cultures du monde ${ }^{19}$. » L'osmose en vue dépasse de loin le peuple noir, ici, puisque Senghor aspirerait à la construction d'un homme universel. Mais sur les bases d'une identité noire d'abord comprise puis assumée et déclarée, avant d'intégrer le reste de l'humanité. Au primat du conflit social se substituerait ici un enjeu sociétal, dans une période marxisante synonyme de lutte des classes et de négociations postcoloniales. Ne peut-on concevoir l'ensemble du discours racialiste (depuis Aristote jusqu'à Tahar Ben Jelloun) comme la longue construction d'une étude de genre: le genre noir? Car si l'on entend par "genre" toute catégorie sociale étudiée à part dans les gender studies anglo-saxonnes (les "études de genre"), l'idée de Noir apparaît comme une catégorie comparable à d'autres telles que la femme, l'immigré, ou l'homosexuel.

L'ébauche d'une nouvelle conscience de soi ne signifie cependant pas qu'il faille changer ce que le noir paraît être: c'est au noir lui-même de changer sa propre façon de se juger et d'apprendre à se regarder tel qu'il est (ou veut être), hors du regard inquisiteur de l'ancien maître blanc. Le philosophe américain Alain Locke (1885-1954) préfigure ce travail de fond avec " Le Nègre Nouveau ». Dans cette contribution aux accents d'homme nouveau, l'auteur dit de l'homme noir à venir qu'il " refuse de souscrire aux jugements traditionnels à son égard, et aux complaisances philanthropiques que cela implique. Il veut être jugé d'une manière objective et rationnelle, rompre

19 Mamadou Diouf : «Les racines retrouvées », in Le Point (Hors Série, avril-mai 2009) : « La pensée noire », p. 45. 
avec sa désillusion sociale, atteindre à une dignité de race, quitter l'état d'esprit d'un débiteur, collaborer à l'œuvre commune et prendre ses responsabilités. » Cet homme se définit dans l'Histoire, puisque la souffrance de la traite détermine son rapport tristement fusionnel avec d'autres peuples. A commencer par le juif, dans la mesure où ce dernier entretient une relation très étroite avec le noir: Garvey s'est inspiré du sionisme pour justifier son panafricanisme; le Juif a subi un traitement similaire au noir dans l'Histoire (Shoah, Code Noir); le Juif représente également un cas de conscience bien à part dans les différentes formes d'expression de la lutte noire: les noirs peuvent-ils condamner les victimes d'une diaspora forcée, tout comme eux-mêmes l'ont toujours été par les blancs? Locke prend le parti inverse et s'associe au juif par solidarité, lui aussi persécuté et considéré pour la peine comme un frère de souffrance:

Comme pour les Juifs, la persécution rend le Nègre international. En tant que phénomène mondial, cette conscience plus large de nous-mêmes (...) était inévitable et ce qui l'a causé, nous ne l'avons pas inventé. Ses conséquences ne sont pas nécessairement préjudiciables aux intérêts supérieurs de la civilisation. Qu'elles provoquent en fait de nouveaux et innombrables conflits, ou au contraire, qu'elles entraînent des échanges culturels et la diffusion de nouvelles lumières, c'est ce que seule peut décider l'attitude des races dominantes, dans notre époque de changement critique ${ }^{20}$.

La prise de distance avec le nationalisme noir est manifeste, ici; distance que finira par prendre aussi Malcolm X vers sa toute fin de vie, lorsqu'il troquera la fierté des siens pour l'amour de tous.

Pieuses paroles, ou initiation à l'homme universel par la négritude? Essayons encore de comprendre cette construction du nouvel homme noir en deux étapes : affirmation de soi, puis intégration de l'autre. Quitte à prendre un raccourci dialectique en la personne de Sartre, très impliqué dans diverses préfaces d'ouvrages sur la négritude.

Les mots de Senghor furent sujets à caution. Et pour cause, lorsque celui-ci déclara que «l'émotion est nègre comme la raison hellène ». L'auteur se justifia dans son cinquième volet de Liberté (sur le dialogue des cultures) par une distinction conceptuelle au sein de la raison :

Je le sais, on m'a reproché d'avoir défini l'émotion comme "nègre" et la raison "héllène", comme européenne si vous voulez (...) Là où la raison discursive, la raison-œil du Blanc, s'arrête aux apparences de l'objet, la raison intuitive, la raison-étreinte du Nègre, par-delà le visible, va jusqu'à la sous-réalité de l'objet pour, au-delà du signe, en saisir le sens. Bien sûr, je simplifie, mais il reste que le Blanc européen est d'abord discursif, le Négro-Africain d'abord intuitif. Il reste que tous les deux sont des hommes de raison; des Homines sapientes, mais pas de la même manière ${ }^{21}$.

Senghor compléta son croquis par une différence de sensibilité ou de rapport au monde entre le noir et le blanc: force vitale, respect des ancêtres morts, fonction cosmique de l'art rendent compte du fameux "art nègre" où l'objectif n'est pas d'imiter les apparences sensibles:

Voilà quelles sont les valeurs fondamentales de la Négritude: un rare don d'émotion, une ontologie existentielle et unitaire, aboutissant, par un surréalisme mystique, à un art engagé et fonctionnel, collectif et actuel, dont le style se caractérise par l'image analogique et le parallélisme asymétrique. Voilà ce que nous apportons au "Rendez-vous du donner et du recevoir", en ce siècle de la Civilisation de l'Universel ${ }^{22}$.

Ne faut-il pas voir dans ce plaidoyer une sorte d'essentialisme à rebours, typique du discours figé des catégories et de la substance aristotéliciennes? Si la raison est conservée pour le noir, elle est non seulement émotive mais consubstantielle à son identité et fait de lui une sorte d'être anhistorique. La fraternité historique suggérée plus tôt par Du Bois disparaît par le fait, si le noir est ce qu'il est partout où il va et quelle que soit l'histoire de sa propre génération. Ne trouve-t-on pas par ailleurs les qualités exprimées par Senghor parmi des activités d'hommes blancs telles que l'art

20 Italiques des auteurs.

21 L. S. SENGHOR, 1993, p. 18.

22 Ibid., p. 23. 
conceptuel du cubisme, la volonté de puissance de Nietzsche ou l'élan vital de Teilhard de Chardin?

Une interprétation plus convaincante du double processus de la négritude est peut-être à trouver chez un philosophe blanc et goy: Jean-Paul Sartre (1905-1980), auteur d'une Réflexion sur la question juive (1946) puis préfacier des négrologues Senghor et Fanon. Par négritude, le philosophe marxiste entend d'abord un double mouvement dialectique d'affirmation de soi et de dépassement de cette affirmation en vue de la réalisation d'une société sans race. Plus simplement et plus subtilement, Sartre présente aussi la négritude comme la "négation de la négation de l'homme noir" et le triomphe mythologique sur le personnage Narcisse. La formule entre guillemets est à lire avec des lunettes d'hégélien: l'homme noir nie sa négation, au sens où il se révolte face au regard du blanc qui l'a toujours réduit à l'état de chose. Mais en bon dialecticien, Sartre ajoute que la loi de double négation ne s'applique pas dans ce cas. En effet, la négation de la négation n'équivaut pas à la simple affirmation car l'homme noir fait plus que s'affirmer lorsqu'il procède de la sorte. Il tient compte d'abord de sa dénégation par l'Autre raciste, puis il dépasse l'état inverse de l'affirmation afin d'atteindre une posture d'homme universel. L'homme noir fait donc plus que s'affirmer par la négritude: il prend conscience de sa condition d'individu racialisé pour s'en abstraire ensuite et garantir ainsi les conditions nécessaires à la réalisation d'une société sans race.

On retrouve plusieurs symptômes des stades de dénégation et d'affirmation successifs dans les littératures blanche et noire incluses. Chez Jean Genêt le maudit, où le regard d'autrui a fait de lui ce qu'autrui voulait qu'il devînt. Le processus de dépassement de la double négation dialectique a été pour ainsi dire raté par Genêt, dès lors que c'est le regard de l'autre qui détermine la conscience de soi et conduit à l'esclavage mental. Problème bien connu de la négritude, et présent dans la littérature afro-américaine. Ainsi Du Bois, qui évoque dans Les Âmes du peuple noir une double conscience servile chez le noir et fait appel à une double métaphore du voile et de la double vue contradictoire :

Après l'Egyptien et l'Indien, le Grec et le Romain, le Teuton et le Mongol, le Noir est une sorte de septième fils, né avec un voile et doué de double vue dans ce monde américain, - un monde qui ne lui concède aucune vraie conscience de soi, mais qui, au contraire, ne le laisse s'apprécier qu'à travers la révélation de l'autre monde. C'est une sensation bizarre, cette conscience dédoublée, ce sentiment de constamment se regarder par les yeux d'un autre, de mesurer son âme à l'aune d'un monde qui vous considère comme un spectacle, avec un amusement teinté de pitié méprisante. Chacun sent constamment sa double nature - un Américain, un Noir; deux âmes, deux pensées, deux luttes irréconciliables; deux idéaux en guerre dans un seul corps noir, que seule sa force inébranlable prévient de la déchirure $^{23}$.

On peut voir chez Du Bois un précurseur du « désir mimétique » de René Girard: de même que je désire ce que l'autre désire parce qu'il le désire, je ne désire pas non plus ce que l'autre ne désire pas; or si mon désir est déterminé par le regard de l'autre, le raciste provoque le rejet de moi-même et la haine de ce que j'incarne. C'est ce retournement contre soi qui vient d'être évoqué dans le cas de la servitude noire, et c'est cette déficience initiale que l'intelligentsia noire a tenté de supprimer par la première étape de l'affirmation de soi contre l'autre. Contre le blanc dominateur, en vue d'échapper à son regard inquisiteur dirigé. Une seconde peau se fait jour, lorsqu'à la première prise de conscience de la négritude sous le regard négatif du discours blanc (le raciste fait le noir, tout l'antisémite de Sartre fait le juif) succède l'affirmation de la négritude par le regard positif de la culture noire. Dans Les damnés de la terre, Frantz Fanon voit dans le processus de décolonisation l'idée d'une construction de soi qui commence avec le regard de l'autre puis finit par s'en libérer sans renier pour autant son rôle historique. Fanon applique ainsi au cas particulier du genre noir le projet existentiel que son préfacier Jean-Paul Sartre associe quant à lui à tout le genre humain: faire en sorte que l'homme noir (le colonisé) soit ce qu'il veuille de ce que les blancs (les colons) ont voulu faire de lui: « La décolonisation est véritablement création d'hommes nouveaux. Mais cette création ne reçoit sa légimité d'aucune puissance surnaturelle: la "chose" colonisée devient homme dans le

23 W. E. B. Du Bors, 2007, p. 11. 
processus même par lequel elle se libère ${ }^{24}$. » Si le noir résulte de sa libération, il est analogue à la femme de Simone Beauvoir: il ne naît pas en tant que tel, mais il le devient par procuration et peut renoncer à ce statut forcé s'il en refuse la responsabilité. Dans le même ordre d'idées, l'écrivain américain James Baldwin (1924-1987) décrivit dans La prochaine fois, le feu la couleur de peau comme construction d'un genre:

Humainement, personnellement la couleur n'existe pas. Politiquement elle existe. Mais c'est là une distinction si subtile que l'Ouest n'a pas encore été capable de la faire. Et au centre de ce terrible orage, de cette vaste confusion se trouvent les Noirs de ce pays à qui il faut maintenant partager le sort d'une nation qui ne les a jamais acceptés et dans laquelle ils furent amenés couverts de chaînes ${ }^{25}$.

Je fus toujours un Autre, lorsque "je” est un noir asservi puis colonisé; la négritude a pour but qu'il en aille autrement.

La seconde étape manifeste le "nouvel humanisme" proposé par la négritude, débarrassé de ses oripeaux européens et ouvert sur un projet universel. Pas un contenu particulier, mais avant tout une posture ou un jeu de dialogues conscient de ce que l'absence de communication peut entraîner dans l'Histoire des peuples. La chose n'est pas simple, comme l'expriment ces vers porteurs de contradiction interne dans le « Cahier d'un retour au pays natal » de Césaire :

Ne faites point de moi cet homme de haine pour qui je n'ai que haine - car pour me cantonner en cette unique race vous savez pourtant mon amour tyrannique - vous savez que ce n'est point par haine des autres races - que je m'exige bêcheur de cette unique race - que ce que je veux - c'est pour la faim universelle - pour la soif universelle ${ }^{26}$.

Le pari de Césaire n'est rien moins que rester noir sans s'y confiner, c'est-à-dire devenir ce que Sartre évoquait à travers sa figure du "Juif authentique": celui qui coexiste avec autrui mais ne vit ni de lui, ni par lui. Une quadrature du cercle que le Martiniquais résuma ainsi : «Je peux changer en échangeant avec l'autre sans me perdre ni me dénaturer ». Mais le processus de créolisation propre à l'Histoire des déportés noirs n'a-t-il pas transformé cette soi-disant nature en question, au point de dénaturer leurs descendants à l'inverse de ce que la négritude prétend? Si l'émotivité mentionnée par Senghor est admise comme un trait typique du noir, Césaire suppose que son histoire s'est mélangée à d'autres expériences et a modifié de fait les racines continentales du peuple africain. Le philosophe, poète et romancier martiniquais Edouard Glissant (1928-2011) semble suivre cette lecture dans son Traité du Tout-Monde, où l'auteur distingue le "Tout-Monde" fluide du "ChaosMonde" brutal de la rencontre coloniale et remplace le modèle simpliste des racines par la structure entremêlée du rhizome (dans la lignée de Deleuze et Guattari).

J'appelle Chaos-monde le choc actuel de tant de cultures qui s'embrassent, se repoussent, disparaissent, subsistent pourtant, s'endorment ou se transforment, lentement ou à vitesse foudroyante : ces éclats, ces éclatements dont nous n'avons pas commencé de saisir le principe ni l'économie et dont nous ne pouvons pas prévoir l'emportement. Le ToutMonde, qui est totalisant, n'est pas (pour nous) idéal. Et j'appelle Poétique de la Relation ce possible de l'imaginaire qui nous porte à concevoir la globalité insaisissable d'un tel Chaos-monde, en même temps qu'il nous permet d'en relever quelque détail, et en particulier de chanter notre lieu, insondable et irréversible. L'imaginaire n'est pas le songe, ni l'évidé de l'illusion ${ }^{27}$.

La touche finale au programme de négritude est d'ordre moral: ne pas commettre soi-même les blessures que l'Autre a infligées plus tôt, mais réaliser tout au contraire une double réconciliation de la catégorie noire avec elle-même puis avec les autres. Cette injonction explique le dépassement de

24 F. FANON, 2011, p. 452.

25 J. BALDWIN, 1963, p. 134.

26 A. CeSAIRE, 1995, p. 50.

27 E. Glissant, 1997, p. 30. 
l'affirmation de soi et légitime l'ouverture à l'Autre, aussi cruel put-il être dans un passé même proche et par opposition au discours de la rupture nationaliste. Car si l'ouverture est possible, elle exige des précautions que la colonisation européenne n'avait pas respectées. En revanche, la négritude assume l'affirmation de soi tout en la distinguant du rejet de l'autre: la première étape d'autodéfense doit laisser place à une ouverture sereine, dès que la conscience affermie permet de faire front et proposer le dialogue avec l'Autre autrefois dominateur. Le rapport de force ne doit pas être renversé, mais supprimé. Dans Peau noire, masques blancs, Frantz Fanon synthétise la facticité du monde et l'existentialisme de Sartre en ces termes, tout autant qu'il incarne le regard transformé de Malcolm X après son pèlerinage de La Mecque. On y trouve une pique à l'actuel thème de la repentance et ses lois mémorielles, ainsi qu'un rejet du rejet de l'autre qui rappelle l'Amour chrétien et donne peut-être sens à l'émotivité substantielle du noir de Senghor:

\begin{abstract}
Vais-je demander à l'homme blanc d'aujourd'hui d'être responsable des négriers du XVIIe siècle ? Vais-je essayer par tous les moyens de faire naître la Culpabilité dans les âmes ? La douleur morale devant la densité du Passé ? Je suis nègre et des tonnes de chaîne, des orages de coups, des fleuves de crachats ruissellent sur mes épaules. Mais je n'ai pas le droit de me laisser ancrer. Je n'ai pas le droit d'admettre la moindre parcelle d'être dans mon existence. Je n'ai pas le droit de me laisser engluer par les déterminations du passé. Je ne suis pas l'esclave de l'Esclavage qui déshumanisa mes pères. Je suis mon propre fondement. Moi, homme de couleur, je ne veux qu'une seule chose : que jamais l'instrument ne domine l'homme. Que cesse à jamais l'asservissement de l'homme par l'homme. C'est-à-dire de moi par un autre. Qu'il me soit permis de découvrir et de vouloir l'homme, où qu'il se trouve. Le nègre n'est pas. Pas plus que le Blanc ${ }^{28}$.
\end{abstract}

\title{
Dialogue III : La lettre par l'esprit (aspect perlocutoire)
}

Le troisième et dernier aspect du discours suppose une participation de la part de l'Autre. Si le locuteur noir a exposé son objectif et ses moyens pour l'obtenir (l'émancipation du regard blanc), qu'en pense l'interlocuteur blanc et dans quelle mesure a-t-elle compris le sens du propos ? Les auteurs de ces lignes sont concernés de près par cette conclusion, puisqu'une parole plus personnelle leur est proposée afin d'illustrer l'aspect perlocutoire du discours sur la négritude.

L'interlocuteur doit comprendre le message qui lui est adressé afin de satisfaire à ses conditions de succès. Mais peut-il souhaiter sincèrement le succès de son vis-à-vis discursif, partenaire autant qu'adversaire potentiel lorsque le noir s'adresse à la communauté de ses bourreaux historiques? Et quand bien même l'interlocuteur ferait preuve de bonne foi, le locuteur initial a-t-il seulement besoin de cette coopération pour servir sa cause ou peut-il imposer son discours par un coup de force symbolique (victoire sur les consciences, discours victimaire ou lacrymal, principe de la repentance)? S'agit-il de convaincre, de persuader, ou de vaincre par tous les moyens?

Concluons notre lecture postraciale par une réponse postmoderne à la question de départ, où la notion de savoir est indissociable de celle de pouvoir et constitue une épreuve de force entre plusieurs dialogues conflictuels. Qui du noir et du blanc produit le discours de la vérité? Impossible de valider l'un ou l'autre projet de civilisation, selon qu'il revendique la défense d'une terre ou prône la fusion par-delà les mers. Si le créole doit sa facticité a son histoire tragique, le blanc vit souvent au sein d'Etats-nations que la puissance et l'héritage glorieux donnent envie de ne garder rien qu'à soi et sans partage. Enchaînement volontaire au passé, culte des morts et souci de l'héritage famial qui maintient une barrière infranchissable avec l'étranger. La première question en appelle une autre, susceptible d'apporter d'autres pistes de réponse au thème du racisme. Qu'est-ce qu'une civilisation? Que doit-elle être? Que peut-elle être? Quelles sont les conditions de sa décadence? C'est au nom de la civilisation que des minorités sont exclues et mettent en évidence le problème d'intégration de certaines catégories, ou genres. Comment concilier majorités et minorités au sein d'une même communauté, et pour quelle raison devrait-on refuser tout refus d'intégration

28 F. FANON, 2011, p. 250-251 (italiques des auteurs). 
vers la globalisation universelle? Si le genre n'est qu'une construction historique, alors la substance n'existe pas et la société est à même de modifier les regards que chacun porte sur l'autre par le jeu de l'éducation. Mais si, dans le en même temps, l'échange n'est avant tout que d'ordre commercial, ${ }^{29}$ alors le discours séparatiste d'un Marcus Garvey vaut bien la quête d'universalité d'un Aimé Césaire et rien d'autre ne les distigue qu'une différence de choix de civilisation. Peut-être faut-il revoir enfin la métaphore du sens, à l'œuvre derrière la notion de "projet de civilisation". S'agit-il d'un sens spatial, comparable à la trajectoire d'une flèche dont le parcours dépend de son point de départ et tend vers une cible (un but, une finalité) précise? Faut-il lui préférer le sens maritime du ressac de Glissant: concevoir une civilisation comme un va-et-vient de cultures entrechoquées, sans finalité prédéfinie?

A bien y regarder, le raciste n'a plus rien de ce fixiste crédule qui associe l'intelligence à la forme du crâne et aux détails physiques. Il est davantage xénophobe, dès lors qu'il rejette l'Autre par crainte de ce que son héritage présent pourrait perdre à le laisser entrer dans son monde. Opposition de styles incarnés par deux archétypes, le Blanc et le Noir. La glèbe et la mer; la cathédrale et le statuaire; civilisation fourre-tout, musée des curiosités, faute de goût ou goût à redéfinir? Notre conclusion ne fait que projeter dans l'avenir et remettre à plus tard la construction de cette cité en mouvement, déformée par le ressac incessant et fondée sur l'imagination au futur plus que sur la raison du passé. Un pari pascalien se cache derrière la négritude, où un peuple soumis à la tragédie incarne l'enjeu du XXI ${ }^{\mathrm{e}}$ siècle: passer du no land's man (diaspora apatride) au no man's land (terre globalisée, village unique sans hommes authentiques, réduits à des agents économiques synonymes de variables d'ajustement)? Glissant a visé juste par son jargon de circonstance: comment éviter que la mondialité des peuples (le Tout-monde) ne se fourvoie en une mondialisation des masses (le Chaos-monde, ou système-empire uniformisé de force)? C'est cet enjeu que la négritude a donné l'occasion de formuler, à défaut d'apporter une réponse manifeste.

La parole finale ne doit être réservée ni à des blancs, ni à des noirs, ni à aucun individu trop conscient de sa couleur et ses implications sur la conscience. Laissons-là aux mots eux-mêmes, puisque l'esprit se conquiert par la lettre tout comme la négritude par la révolte. Au commencement était le verbe ...

I am not a dancer. I am a dance floor. I am not your brother. I am a son. I am not a writer. I am a page.

(Anonyme)

\section{Ouvrages cités}

BALDWIN, J., 1963, La prochaine fois, le feu, trad. M. Sciama, Gallimard.

Cesaire, A., 1995, « Cahier d'un retour au pays natal » (1956), in Présence Africaine.

Carmichael M. \& Hamilton C. V., 1968, Black Power. Pour une politique de libération aux Etats-Unis (1967), trad. Odile Pidoux, Collection Payot.

Douglass, F., 2007, Mémoires d'un esclave (1845), trad. Chantal Santerre et Normand Baillargeon, Lux (éd.), Montréal, Collection « Mémoire des Amériques ».

29 L'exemple du sport est emblématique d'une créolisation du monde occidental sur fond de libre-échange: le football et le basket ont largement contribué à l'enrichissement de la population noire, substituant pour ainsi dire les anciennes chaînes en fer autour des pieds par de nouvelles chaînes en or autour du cou. Effet secondaire de la décolonisation, cet aspect de la créolisation caractérise bien plus le Chaos-Monde de Glissant que son idéal du ToutMonde. 
Du BoIs, W. E. B., 2007, Les Âmes du peuple noir (1903), trad. M. Bessone, Collection Versions Françaises, Editions Rue d'Ulm.

FANON, F., 2011, Oeuvres. Editions La Découverte, Paris.

Glissant, E., 1997, Traité du Tout-Monde (Poétique IV), Gallimard.

LOCKE, A., $193 \quad$ 1, « Le Nègre nouveau », trad. Renée et Louis Guilloux, in Europe, n¹02.

McKay, C., 1999, Banjo (1928), trad. Michel Fabre, Editions André Dimanche.

PRICE-MARS, J., 2009, Ainsi parla l'oncle (1928), Mémoire d'encrier, Montréal.

SARTRE, J.-P., 1954, « Réflexions sur la question juive » (1946), Folio Essais, Gallimard.

SARTRE, J.-P., 1948, « Orphée Noir » (1948), Préface de l'Anthologie de la nouvelle poésie nègre et malgache de langue française (L. S. Senghor).

Senghor, L. S., 1993, Liberté V: le Dialogue des cultures, Seuil.

WalcotT, D., 1998, Le royaume du fruit-étoile, trad. Claire Malroux, Editions Circé. 